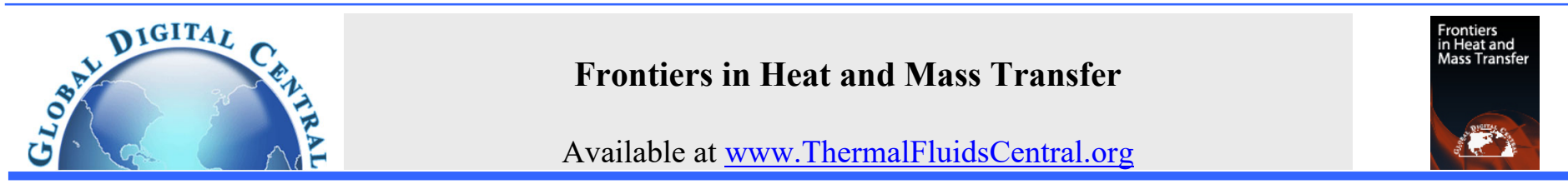

\title{
SEEBECK EFFECT IN GENERALIZED MAGNETO-THERMO VISCOELASTIC SPHERICAL REGION
}

\author{
Alaa K. Khamis ${ }^{\text {a }}$, Allal Bakali a ${ }^{\text {, A. A. El-Bary }}{ }^{\text {b, } c^{*}}$, Haitham M. Atef ${ }^{\text {d }}$ \\ ${ }^{a}$ Faculty of Science, Northern Border University, Arar, Kingdom of Saudi Arabia \\ ${ }^{b}$ Arab Academy for Science, Technology and Maritime Transport, P.O. Box 1029, Alexandria, Egypt \\ ${ }^{c}$ National Committee for Mathematics, Academy of Scientific Research and Technology, Egypt. \\ ${ }^{d}$ Department of Mathematics, Faculty of science, Damanhur University, Damanhur, Egypt
}

\begin{abstract}
In this paper the effect of the magnetic field and Seebeck parameter was investigated. Modified Ohm's law that includes effects of the temperature gradient (Seebeck effect $k_{o}$ ) and charge density, as well as generalized Fourier's law with current density, the problem of conveyance of thermal stresses and temperature in a generalized Magneto-Thermo-Viscoelastic spherical region. The formulation is applied to the generalized thermo visco elasticity dependent on the Green-Naghdi (G-N II) hypothesis, where there is an underlying magnetic field corresponding to the plane limit, because of the utilization of the magnetic field, it results an actuated magnetic and electric fields in the medium. The Laplace change system is utilized to solve the problem. The State Space investigation is applied to acquire the temperature, displacement, stresses, induced electric field, instigated magnetic field and current density. Application is utilized to our concern to get the arrangement in the total structure. The considered variables are introduced graphically and discussions are made.
\end{abstract}

Keywords: Thermal stress; Generalized Magneto-Thermo-Viscoelastic; Spherical Cavity; Seebeck coefficient; Green-Naghdi theory; Thermal shock.

\section{INTRODUCTION}

The traditional uncoupled hypothesis of thermoelasticity predicts two marvels not good with physical perceptions. In the first place, the condition of heat conduction of this hypothesis doesn't contain any elastic terms; second, the heat condition is of a parabolic kind, anticipating unending paces of spread for heat waves. Biot (1956) presented the hypothesis of coupled thermoelasticity to conquer the principal weakness. The overseeing conditions for this hypothesis are coupled, dispensing with the main oddity of the old style hypothesis. In any case, the two hypotheses share the second inadequacy since the heat equation for the coupled hypothesis is likewise parabolic.

Two generalizations to the coupled hypothesis were presented. The first is because of Lord and Shulman (1976), who acquired a wave-type heat equation by proposing another law of heat equation to supplant the old style Fourier's law. Since the heat equation of this hypothesis is of the wave type, it consequently guarantees limited velocities of spread for heat and elastic waves. The staying administering equations for this hypothesis, to be specific, the equations of motion and constitutive relations, continue as before as those for the coupled and the uncoupled speculations. The second speculation to the coupled hypothesis of elasticity is what is known as the hypothesis of thermo elasticity with two relaxation times or the hypothesis of temperature-rate-dependent thermo elasticity. Müller (1976), in a survey of the thermodynamics of thermo elasticity solids, proposed an entropy creation imbalance, with the assistance of which he thought about limitations on a class of constitutive equations. A generalization of this imbalance was proposed by Green and Laws (1972). Green and Lindsay got an express form of the constitutive equations in (1972). These equations were additionally gotten autonomously by Suhubi (1957) has acquired the fundamental solution for this hypothesis. This hypothesis contains two constants that go about as relaxation times and alter all the equation of the coupled hypothesis, not just the heat equation. The old style Fourier's law of heat equation isn't disregarded if the medium viable has a focal point of balance.

Later Green and Naghdi (1991), (1992) and (1993) proposed three hypotheses of generalized thermo elasticity. The primary model (G-N I) is actually equivalent to Biot's hypothesis (1956). The second and third models are named as G-N II and G-N III model. In G-N II and G-N III models, the thermal wave engenders with limited rates which concur with physical circumstances. A significant component of G-N II hypothesis is that this hypothesis doesn't suits dissipation of thermal energy though G$\mathrm{N}$ III hypothesis obliges dissemination of dissipation of thermal energy. With the quick advancement of polymer science and plastic industry, just as the wide utilization of materials under high temperature in present day innovation and use of science and topography in designing, the hypothetical examination and applications in viscoelastic material has become a significant errand for strong mechanics.

The hypothesis of thermo-viscoelasticity and the solutions of some boundary value problems of thermo-viscoelasticity were researched by Illyushin's and Pobedria (1970). Crafted by Biot (1954) and (1955), Morland and Lee (1960) and Tanner (1988) mate extraordinary walks in the most recent decade in discovering answers for limit esteem issues for linear viscoelasticity materials including temperature varieties for both semi static and dynamic issues. Drozdov (1996) inferred a constitutive

\footnotetext{
*Corresponding author.Email: aaelbary@aast.edu
} 
model in thermo-viscoelasticity which represents changes in elastic moduli and relaxation times. Bland (1960) connected the arrangement of linear viscoelasticity issues to comparing linear elastic solutions. Remarkable works right now crafted by Gurtin and Sternberg (1962). Consequence of significant examinations deciding the mechanical properties of viscoelastic material were included by Koltunov's (1976).

The hypothesis of electro-magneto-thermo-viscoelasticity has stimulated a lot of enthusiasm for some mechanical applications especially in atomic gadget. Where there exists an essential magnetic field. Different examinations have been completed by thinking about the connection between attractive, magnetic, thermal and strain fields. Examinations of such issue additionally impact different applications in biomedical building just as in various geometric investigations. Amin et al. (2018) studied the effect of viscous fractional parameter on generalized magneto thermo-viscoelastic thin slim strip exposed to moving heat source. Ismail et al. (2020) discussed the generalized magneto- thermoelasticity and heat conduction on an infinite medium with spherical cavity.

\section{BASIC GOVERNING EQUATIONS}

We shall consider a homogeneous isotropic thermo-viscoelastic medium occupying the region $R \ll r<\infty$ of a perfect electrically conductivity permeated by an initial constant magnetic field $H_{o}$, where $R$ is the radius of the shell.

Due to the effect of this magnetic field there arises in the conducting medium an induced magnetic field $\boldsymbol{h}$ and induced electric field $\boldsymbol{E}$. Also, there arises a force $\boldsymbol{F}$ (the Lorentz Force). Due to the effect of this force, points of the medium undergo a displacement $u$, which gives rise to a temperature.

The linearized equations of electromagnetism for slowly moving media are:

$$
\begin{aligned}
& \text { curl } \boldsymbol{h}=\boldsymbol{J}+\varepsilon_{o} \frac{\partial E}{\partial t}, \\
& \operatorname{curl} \boldsymbol{E}=-\boldsymbol{\mu}_{\boldsymbol{o}} \frac{\partial E}{\partial \boldsymbol{t}}, \\
& B=\mu_{o} H, \\
& \operatorname{div} B=0,
\end{aligned}
$$

The above field equations are supplemented by constitutive equations which consist first of modified ohm's law:

$$
E=-\mu_{o} \frac{\partial u}{\partial t} \times H_{o}+k_{o} \operatorname{grad} \theta
$$

The second constitutive equation is the one for the Lorenz force which is

$$
F=J \times B \text {. }
$$

The third constitutive equation is the stress - displacement temperature relation for viscoelastic medium of Kelvin - Voigt type:

$$
\begin{aligned}
& \sigma_{i j}=2 \mu_{e}\left(1+\alpha_{1} \frac{\partial}{\partial t}\right) e_{i j}+\lambda_{e}\left(1+\alpha_{o} \frac{\partial}{\partial t}\right) e \delta_{i j}- \\
& \gamma_{e}\left(1+\gamma_{o} \frac{\partial}{\partial t}\right) \theta \delta_{i j},
\end{aligned}
$$

The equation of motion is given by:

$$
\begin{aligned}
& \quad \rho \frac{\partial^{2} u_{i}}{\partial^{2} t}=\left(2 \mu_{e}\left(1+\alpha_{1} \frac{\partial}{\partial t}\right)+\lambda_{e}\left(1+\alpha_{o} \frac{\partial}{\partial t}\right)\right) u_{j, i j}-\gamma_{e}(1+ \\
& \left.\gamma_{o} \frac{\partial}{\partial t}\right) \theta_{, i}+\mu_{o}\left(J \times H_{o}\right)
\end{aligned}
$$

The generalized heat conduction equation is given by

$$
K \theta_{, i i}+K^{*} \dot{\theta_{, l l}}=\rho C_{E} \dot{\theta}+\gamma_{e} T_{o}\left(1+\gamma_{o} \frac{\partial}{\partial t}\right) \ddot{e}+\pi_{o} \operatorname{div} J
$$

The strain displacement relation is given by

$$
e_{i j}=\frac{1}{2}\left(u_{i, j}+u_{j, i}\right)
$$

Together with the previous equations, constitute a complete system of generalized -magneto-thermo-viscoelasticity equations for a medium with a perfect conductivity.

Let $(r, \psi, \phi)$ denote the radial coordinates, the co- latitude, and the longitude of a spherical coordinates system, respectively. Due to spherical symmetry, all the considered function will be functions of $r$ and $t$ only.
The components of the displacement vector will be taken the form:

$u_{r}=u(r, t), u_{\psi}=u_{\phi}=0$

The strain tensor components are thus given by

$e_{r r}=\frac{\partial u}{\partial r}, e_{\psi \psi}=e_{\phi \phi}=\frac{u}{r}, e_{r \phi}=e_{\phi \psi}=0$

$e=\frac{\partial u}{\partial r}+\frac{2 u}{r}=\frac{1}{r^{2}} \frac{\partial\left(r^{2} u\right)}{\partial r}$

From equation (7) we obtain the components of the stress tensor as

$\sigma_{r r}=2 \mu_{e}\left(1+\alpha_{1} \frac{\partial}{\partial t}\right) \frac{\partial u}{\partial r}+\lambda_{e}\left(1+\alpha_{o} \frac{\partial}{\partial t}\right) e-\gamma_{e}\left(1+\gamma_{o} \frac{\partial}{\partial t}\right) \theta$

$\sigma_{\phi \phi}=\sigma_{\psi \psi}=2 \mu_{e}\left(1+\alpha_{1} \frac{\partial}{\partial t}\right) \frac{u}{r}+\lambda_{e}\left(1+\alpha_{o} \frac{\partial}{\partial t}\right) e-\gamma_{e}\left(1+\gamma_{o} \frac{\partial}{\partial t}\right) \theta$

$\sigma_{r \phi}=\sigma_{r \psi}=\sigma_{\psi \phi}=0$

Assume now that the initial magnetic field acts in the $\phi$-direction and has the components $\left(0,0, H_{o}\right)$.

The induced magnetic field $\mathrm{h}$ will have one component $\mathrm{h}$ in the $\phi-$ direction, while the induced electric field $\mathrm{E}$ will have one component $\mathrm{E}$ in the $\psi$-direction.

Then, equations (1), (2) and (5) yield

$J=H_{o} \frac{\partial e}{\partial r}+\frac{k_{o}}{\mu_{o}} \frac{\partial \theta}{\partial r}$,

$h=-H_{o}\left(\frac{\partial u}{\partial r}+\frac{u}{r}\right)-\frac{k_{o}}{\mu_{o}} \frac{\partial \theta}{\partial r}$,

$E=\mu_{o} H_{o} \frac{\partial u}{\partial t}+k_{o} \frac{\partial \theta}{\partial r}$.

From equations (17) and (6), we get that the Lorentz force has only one component $F_{r}$ in the $r$-direction:

$F_{r}=\mu_{o} H_{o}^{2} \frac{\partial e}{\partial r}+k_{o} H_{o} \frac{\partial \theta}{\partial r}$

Also, we arrived at

$\rho \frac{\partial^{2} u}{\partial t^{2}}=\left(2 \mu_{e}\left(1+\alpha_{1} \frac{\partial}{\partial t}\right)+2 \lambda_{e}\left(1+\alpha_{o} \frac{\partial}{\partial t}\right)+\mu_{o} H_{o}^{2}\right) \frac{\partial e}{\partial r}-\gamma_{e}(1+$

$\left.\gamma_{o} \frac{\partial}{\partial t}\right) \frac{\partial e}{\partial r}+k_{o} H_{o} \frac{\partial \theta}{\partial r}$

Equation (21) is to be supplemented by the constitutive equation (13) and the heat conduction equation

$K \nabla^{2} \theta+K^{*} \nabla^{2} \dot{\theta}=\rho C_{E} \ddot{\theta}+\gamma_{e} T_{o}\left(1+\gamma_{o} \frac{\partial}{\partial t}\right) \ddot{e}+\pi_{o} d i v J$,

where $\nabla^{2}$ is Laplace's operator in spherical coordinates which is given by $\nabla^{2}=\frac{1}{r^{2}} \frac{\partial}{\partial r}\left(r^{2} \frac{\partial}{\partial r}\right)+\frac{1}{r^{2} \sin \psi}\left(\sin \psi \frac{\partial}{\partial \psi}\right)+\frac{1}{r^{2} \sin ^{2} \psi} \frac{\partial^{2}}{\partial \phi^{2}}$

In case of dependence on $r$ only, this reduce to

$\nabla^{2}=\frac{1}{r^{2}} \frac{\partial}{\partial r}\left(r^{2} \frac{\partial}{\partial r}\right)$

Now, we shall use the following non dimensional variables:

$$
\dot{r}=C_{1} \eta r, \dot{u}=C_{1} \eta u, \dot{t}=C_{1}^{2} \eta t, \gamma_{o}^{\prime}=C_{1}^{2} \eta \gamma_{o}, \alpha_{o}=C_{1}^{2} \eta \alpha_{o}, \alpha_{1}^{\prime}=C_{1}^{2} \eta \alpha_{1} \text {, }
$$$$
\sigma_{\imath j}^{\prime}=\frac{\sigma_{i j}}{\mu_{e}}, \dot{\theta}=\frac{\theta}{T_{o}}, \hat{h}=\frac{h}{H_{o}}, \dot{E}=\frac{E}{\mu_{o} H_{o} C 1}, j=\frac{J}{\eta H_{o} C 1}
$$

Equations (14) - (19), (21) and (22) take the following form (dropping the primes for convenience).

$$
\begin{gathered}
J=\frac{\partial e}{\partial r}+A \frac{\partial \theta}{\partial r} \\
h=-\left(\frac{\partial u}{\partial r}+\frac{u}{r}\right)-A \frac{\partial \theta}{\partial r}, \\
E=\frac{\partial u}{\partial t}+A \frac{\partial \theta}{\partial r}, \\
\sigma_{r r}=\frac{2 \mu_{e}}{\lambda_{e}+2 \mu_{e}}\left(1+\alpha_{1} \frac{\partial}{\partial t}\right) \frac{\partial u}{\partial r}+\frac{\lambda_{e}}{\lambda_{e}+2 \mu_{e}}\left(1+\alpha_{o} \frac{\partial}{\partial t}\right) e \frac{\gamma_{e} \theta_{o}}{\lambda_{e}+2 \mu_{e}}(1+ \\
\left.\gamma_{o} \frac{\partial}{\partial t}\right) \theta \\
\sigma_{\phi \phi}=\sigma_{\psi \psi}=\frac{2 \mu_{e}}{\lambda_{e}+2 \mu_{e}}\left(1+\alpha_{1} \frac{\partial}{\partial t}\right) \frac{u}{r}+\frac{\lambda_{e}}{\lambda_{e}+2 \mu_{e}}\left(1+\alpha_{o} \frac{\partial}{\partial t}\right) e- \\
\frac{\gamma_{e} \theta_{o}}{\lambda_{e}+2 \mu_{e}}\left(1+\gamma_{o} \frac{\partial}{\partial t}\right) \theta \\
(27) \\
\sigma_{r \phi}=\sigma_{r \psi}=\sigma_{\phi \psi}=0
\end{gathered}
$$$$
\frac{\gamma_{e} \theta_{o}}{\lambda_{e}+2 \mu_{e}}\left(1+\gamma_{o} \frac{\partial}{\partial t}\right) \theta
$$

$\left.\gamma_{o} \frac{\partial}{\partial t}\right) \frac{\partial \theta}{\partial r}$ 


$$
\nabla^{2} \theta+\frac{K^{*} C_{1}^{2} \eta}{K} \nabla^{2} \dot{\theta}=\eta C_{1}^{2} \ddot{\theta}+\frac{\gamma_{e} C_{1}^{2}}{K}\left(1+\gamma_{o} \frac{\partial}{\partial t}\right) \ddot{e} .
$$

\section{Where,}

$$
\eta=\frac{\rho C_{E}}{K}, C_{1}^{2}=\frac{\lambda_{e}+2 \mu_{e}}{\rho}, A=\frac{k_{o}}{\mu_{o} H_{o}}
$$

Equation (23) can be written in the form:

$$
e=\left(1+A+\mu_{o} H_{o}^{2}+\frac{\lambda_{e} \alpha_{o}+2 \mu_{e} \alpha_{1}}{\rho C_{1}^{2}} \frac{\partial}{\partial t}\right) \nabla^{2} e-\frac{\gamma_{e} \theta_{o}}{\rho C_{1}^{2}}\left(1+A+\gamma_{o} \frac{\partial}{\partial t}\right) \nabla^{2} \theta
$$

\section{LAPLACE TRANSFORM DOMAIN}

Taking the Laplace transform of equations (23)-(31) by using homogeneous initial conditions, defined and denoted as

$\bar{f}(s) \int_{0}^{\infty} e^{-s t} f(t) d t \quad, s>0$

We obtain

$\bar{J}=\frac{d \bar{e}}{d r}+A \frac{d \bar{\theta}}{d r}$

$\bar{h}=-\left(\frac{d \bar{u}}{d r}+\frac{\bar{u}}{r}\right)-A \frac{d \bar{\theta}}{d r}$

$\bar{E}=s \bar{u}+A \frac{d \bar{\theta}}{d r}$

$\nabla^{2} \bar{\theta}=L_{1} \bar{\theta}+L_{1} \bar{e}$

$\bar{\sigma}_{r r}=a_{1} \frac{d \bar{u}}{d r}+a_{2} \bar{e}-a_{3} \bar{\theta}$,

$\bar{\sigma}_{\phi \phi}=\bar{\sigma}_{\theta \theta}=a_{1} \frac{\bar{u}}{r}+a_{2} \bar{e}-a_{3} \bar{\theta}$

$\nabla^{2} \bar{e}=M_{1} \bar{\theta}+M_{1} \bar{e}$

Where

$L_{1}=\frac{K \eta C_{1}^{2} s^{2}}{K+A+K^{*} \eta C_{1}^{2} s^{2}}, l_{2}=\frac{\gamma_{e} C_{1}^{2}\left(1+\gamma_{o} s\right) s^{2}}{K+A+K^{*} \eta C_{1}^{2} s^{2}}$

$, a_{1}=\frac{2 \mu_{e}}{\lambda_{e}+2 \mu_{e}}\left(1+A+\alpha_{1} s\right), a_{2}=\frac{\lambda_{e}}{\lambda_{e}+2 \mu_{e}}\left(1+A+\alpha_{o} s\right)$

$a_{3}=\frac{\gamma_{e} \theta_{o}}{\lambda_{e}+2 \mu_{e}}\left(1++A \gamma_{o} s\right)$,

$$
M_{1}=\frac{\gamma_{e} \theta_{o}\left(1++A \gamma_{o} s\right) L_{1}}{\mu_{o} H_{o}^{2} \rho C_{1}^{2}+\rho C_{1}^{2}+\left(\lambda_{e} \alpha_{o}+2 \mu_{e} \alpha_{1}\right) s},
$$

,$M_{2}=\frac{\rho C_{1}^{2} s^{2}+\gamma_{e} \theta_{o}\left(1++A \gamma_{o} s\right) L_{2}}{\mu_{o} H_{o}^{2} \rho C_{1}^{2}+\rho C_{1}^{2}+\left(\lambda_{e} \alpha_{o}+2 \mu_{e} \alpha_{1}\right) s}$

\section{STATE SPACE FORMULATION}

Choosing as state variables the temperature of heat conduction $\bar{\theta}$ and the strain components $\bar{e}$ then equations (35) and (38) can be written in the matrix form

$\nabla^{2} V(r, s)=A(s) \bar{V}(r, s)$

$\bar{V}(r, s)=\left[\begin{array}{l}\bar{\theta}(r, s) \\ \bar{e}(r, s)\end{array}\right], A(s)=\left[\begin{array}{ll}L_{1} & L_{2} \\ M_{1} & M_{2}\end{array}\right]$

The formal solution of system (39) can be written in the form

$\bar{V}(r, s)=C \frac{e^{-\sqrt{A(s)} r}}{r}+B \frac{e^{-\sqrt{A(s)} r}}{r}$

For bounded solution with large $r$, we have canceled the exponential part has positive power. And at $r=R$ the value of $C$ is given by

$C=R \bar{V}(R, s) e^{\sqrt{A(s)} R}$, then equation (40) reduces to

$\bar{V}(r, s)=\frac{R}{r} \bar{V}(R, s) e^{-\sqrt{A(s)} R} \quad, r \geq R$

We will use the will-known Cayley-Hamiltonian theorem to find the form of the matrix

$\exp (-\sqrt{A(s)}(r-R))$

The characteristic equation of the matrix $A(s)$ can be written as
$k^{2}-\left(L_{1}+M_{2}\right) k+\left(L_{1} M_{2}-L_{2} M_{1}\right)$

$(4$

The roots of this equation namely $k_{1}$ and $k_{2}$ satisfy the relations

$k_{1}+k_{2}=L_{1}+M_{2}$

$k_{1} k_{2}=L_{1} M_{2}-L_{2} M_{1}$

The Tailor's series expansion for the matrix exponential of

$\exp (-\sqrt{A(s)}(r-R))$ is given by

$\exp (-\sqrt{A(s)}(r-R))=\sum_{n=0}^{\infty} \frac{[-\sqrt{A(s)}(r-R)]^{n}}{n !}$

Using Cayley - Hamiltonian theorem, we can express $A^{2}$ and higher orders of the matrix $A$ in terms of $I$ and $A$

where $I$ is the unit matrix of second order.

Thus, the infinite series in equation (42) can be reduced to

$\exp (-\sqrt{A(s)}(r-R))=b_{o}(r, s) I+b_{1}(r, s) A$

Where $b_{o}$ and $b_{1}$ are some coefficients depending on $s$ and $r$.

By Cayley- Hamiltonian theorem, the characteristic roots $k_{1}$ and

$k_{2}$ of the matrix $A$ must satisfy equation (42), thus we have

$\exp \left(-\sqrt{k_{1}}(r-R)\right)=b_{o}+b_{1} k_{1}$

$\exp \left(-\sqrt{k_{2}}(r-R)\right)=b_{o}+b_{1} k_{2}$

Solving the above linear system of equations, we get

$b_{o}=\frac{k_{1} e^{-\sqrt{k_{2}}(r-R)}-k_{2} e^{-\sqrt{k_{1}}(r-R)}}{k_{1}-k_{2}}$

$b_{1}=\frac{e^{-\sqrt{k_{1}}(r-R)}-e^{-\sqrt{k_{2}}(r-R)}}{k_{1}-k_{2}}$

Hence, we have

$\exp (-\sqrt{A(s)}(r-R))=L_{i j}(r, s), \quad i, j=1,2$

Where,

$L_{11}=\frac{e^{-\sqrt{k_{2}}(r-R)}\left(k_{1}-L_{1}\right)+e^{-\sqrt{k_{1}}(r-R)}\left(L_{1}-k_{2}\right)}{k_{1}-k_{2}}$

$L_{12}=\frac{L_{2} e^{-\sqrt{k_{1}}(r-R)}-L_{1} e^{-\sqrt{k_{2}}(r-R)}}{k_{1}-k_{2}}$

$L_{21}=\frac{M_{1} e^{-\sqrt{k_{1}}(r-R)}-M_{2} e^{-\sqrt{k_{1}}(r-R)}}{k_{1}-k_{2}}$

$L_{22}=\frac{e^{-\sqrt{k_{1}}(r-R)}\left(M_{2}-k_{2}\right)+e^{-\sqrt{k_{2}}(r-R)}\left(k_{1}-M_{2}\right)}{k_{1}-k_{2}}$

\section{APPLICATIONS}

In order to evaluate the unknown parameters $\bar{\theta}_{o}(r, s)$ and $\bar{e}_{o}(r, s)$, we shall use the boundary conditions on the internal surface of the shell, $r=$ $R$ which are given by:

(I) Thermal boundary condition at $r=R$

$\theta(R, t)=\theta_{o}$

Taking the Laplace transform, this is defined as following:

$\bar{\theta}_{o}(r, s)=\frac{\theta_{o}}{s}$

\section{(II) Mechanical boundary condition}

The internal surface $r=R$ has a rigid foundation, which is rigid enough to prevent any strain

$e(R, t)=0$

Taking the Laplace transform, this is defined as following:

$\bar{e}(R, s)=\bar{e}_{o}=0$

Using the conditions (49) and (50) into equation (34) and using equations (44)-(48), we get

$$
\begin{aligned}
& \bar{\theta}(r, s)=\frac{R \theta_{o}}{s\left(k_{1}-k_{2}\right) r}\left[\left(k_{1}-L_{2}\right) e^{-\sqrt{k_{2}}(r-R)}+\left(k_{2}-L_{1}\right) e^{-\sqrt{k_{2}}(r-R)}\right] \\
& \bar{e}(r, s)=\frac{R M_{1} \theta_{o}}{s\left(k_{1}-k_{2}\right) r}\left[\left(k_{2}-L_{1}\right) e^{-\sqrt{k_{1}}(r-R)}+\left(k_{1}-L_{2}\right) e^{-\sqrt{k_{2}}(r-R)}\right]
\end{aligned}
$$


To find the displacement, taking Laplace transform for equation (23) and using equations (51) and (52), we get

$\bar{u}(r, s)=\frac{R \theta_{o}}{s r^{2}\left(k_{1}-k_{2}\right) r}\left(\left(\left(1+r \sqrt{k_{1}}\right)\left(B_{5}\left(L_{1}-k_{2}\right)\right)-\right.\right.$

$\left.B_{4} M_{1}\right) e^{-\sqrt{k_{1}}(r-R)}+\left(\left(1+r \sqrt{k_{2}}\right)\left(B_{4} M_{1}\right.\right.$
$\left.\left.\left.\left.L_{1}\right)\right)\right) e^{-\sqrt{k_{2}}(r-R)}\right)$

To find the stresses, from equations (36), (37) and (58)- (60) we get

$\bar{\sigma}_{r r}=\frac{R \theta_{o}}{s r^{2}\left(k_{1}-k_{2}\right) r}\left\{e^{-\sqrt{k_{1}}(r-R)}\left(M_{1}\left(B_{1}+B_{3}\right) r^{2}+2 B_{1} B_{4} M_{1}(1+\right.\right.$

$\left.\left.\left.r \sqrt{k_{1}}\right)-\left(L_{1}-k_{2}\right)\left(B_{3} r^{2}+2 B_{1} B_{5}\left(1+r \sqrt{k_{1}}\right)\right)\right)+e^{-\sqrt{k_{2}}(r-R)}\right\}$

where,

$B_{4}=\frac{1}{s^{2}}+\frac{\lambda_{e} \alpha_{o}+2 \mu_{e} \alpha_{1}}{\rho C_{1}^{2} s^{2}}$

$B_{5}=\frac{\gamma_{e} \theta_{o}\left(1+\gamma_{o} s\right)}{\rho C_{1}^{2} s^{2}}$

\section{NUMERICAL INVERSION OF LAPLACE TRANSFORMS}

In order to invert the Laplace transforms in the above equations we shall use a numerical technique based on Fourier expansions of functions. Let $\bar{g}(s)$ be the Laplace transform of a given function $g(t)$. The inversion formula of Laplace transforms states that

$g(t)=\frac{1}{2 \pi i} \int_{d-i \infty}^{d+i \infty} e^{s t} \bar{g}(s) d s$

Where $d$ is an arbitrary positive constant greater than all the real parts of the singularities of $\bar{g}(s)$. Takings $=d+i y$, we get

$g(t)=\frac{e^{d t}}{2 \pi} \int_{-\infty}^{\infty} e^{i k y} \bar{g}(d+i y) d y$

This integral can be approximated by

$g(t)=\frac{e^{d t}}{2 \pi} \sum_{k=-\infty}^{\infty} e^{i k t \Delta y} \bar{g}(d+i k \Delta y) \Delta y$

Taking $\Delta y=\frac{\pi}{t_{1}}$ we obtain:

$g(t)=\frac{e^{d t}}{t_{1}}\left(\frac{1}{2} \bar{g}(d)+\operatorname{Re}\left(\sum_{k=1}^{\infty} e^{i k t \pi / t_{1}} \bar{g}\left(d+i k \pi / t_{1}\right)\right)\right)$

For numerical purposes this is approximated by the function $g_{N}(t)=\frac{e^{d t}}{t_{1}}\left(\frac{1}{2} \bar{g}(d)+\operatorname{Re}\left(\sum_{k=1}^{N} e^{i k t \pi / t_{1}} \bar{g}\left(d+i k \pi / t_{1}\right)\right)\right)$

(63)

Where $N$ is a sufficiently large integer chosen such that

$\frac{e^{d t}}{t_{1}} \operatorname{Re}\left(e^{i N \pi t / t_{1}} \bar{g}\left(d+i N \pi / t_{1}\right)\right)<\eta$

Where $\eta$ is a reselected small positive number that corresponds to the degree of accuracy to be achieved Formula (49) is the numerical inversion formula valid for $0 \leq t \leq t_{1}$. In particular, we chooset $=t_{1}$, getting

$g_{N}(t)=\frac{e^{d t}}{t_{1}}\left(\frac{1}{2} \bar{g}(d)+R e\left(\sum_{k=1}^{N}(-1)^{k} \bar{g}\left(d+i k \pi / t_{1}\right)\right)\right)$

\section{NUMERICAL RESULTS AND DISCUSSIONS}

The copper material was chosen for purposes of numerical evaluations and constants of the problem were taken as following $K=386 \mathrm{~N} / \mathrm{Ks}, \alpha_{t}=17.8(10)^{-5} \mathrm{~K}^{-1}, C_{E}=383.1 \mathrm{~m}^{2} / \mathrm{K}$ , $T_{o}=293 \mathrm{~K}, \rho=8954 \mathrm{~kg} / \mathrm{m}^{2}$

$$
, \alpha_{o}=6.8831(10)^{-13}, \alpha_{o}=6.8831(10)^{-13}, R=1, \theta_{o}=1
$$

In order to study the effect of time $t$ and study the comparison between two models on temperature, radial stress, shear stress, displacement and strain, we now present our results in the form of graphs (Figs. 1-8).

Fig. 1 is plotted to show the variation of temperature $\theta$ against $r$ for wide range of $r(1 \leq r \leq 3)$ at small time $(t=0.07)$ for two theories (G-N III) and (G-N II) It is observed from this figure the magnitude of the temperature is greater for (G-N III) model than (G-N II). It can be noted that the speed of propagation of temperature is finite and coincide with the physical behavior of viscoelastic material. Also, we can see from this figure that the boundary condition (56) is satisfied.

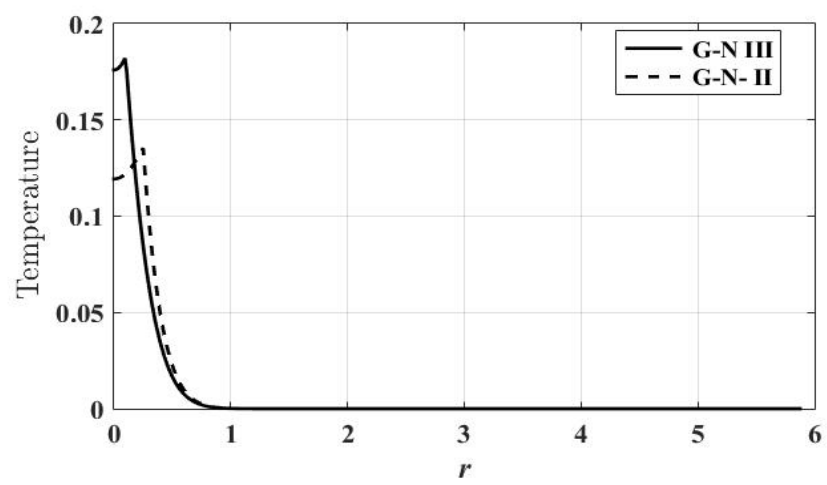

Fig. 1 Variation of Temperature $\theta$ with distance $r$ for two theories

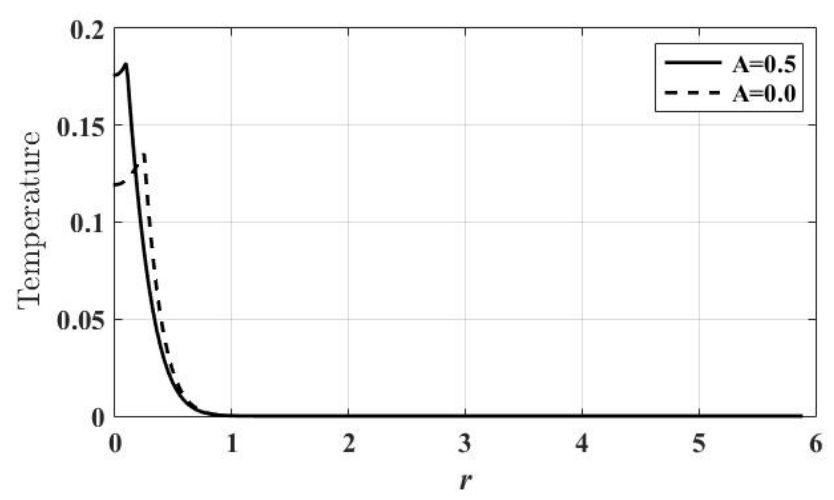

Fig. 2 Variation of Temperature $\theta$ with distance $r$ for the coefficient of Ohm and Fourier laws

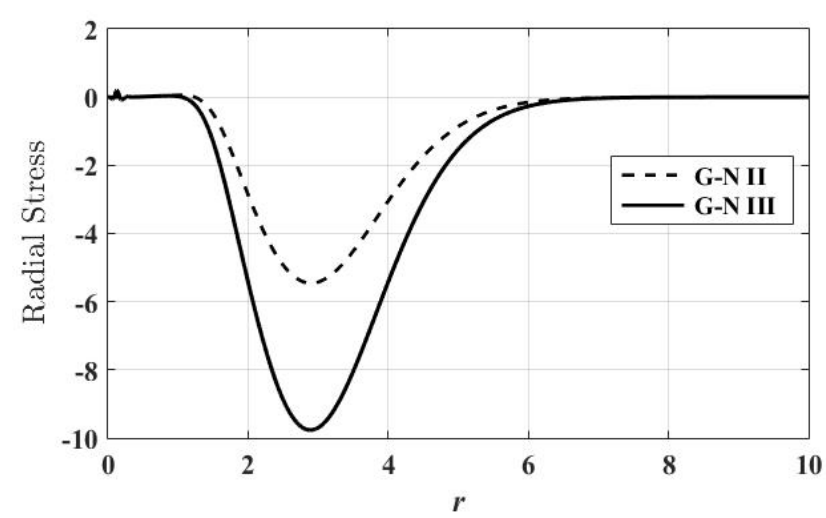

Fig. 3 Variation of Radial Stress $\sigma_{r r}$ with distance $\mathrm{r}$ for two theories 


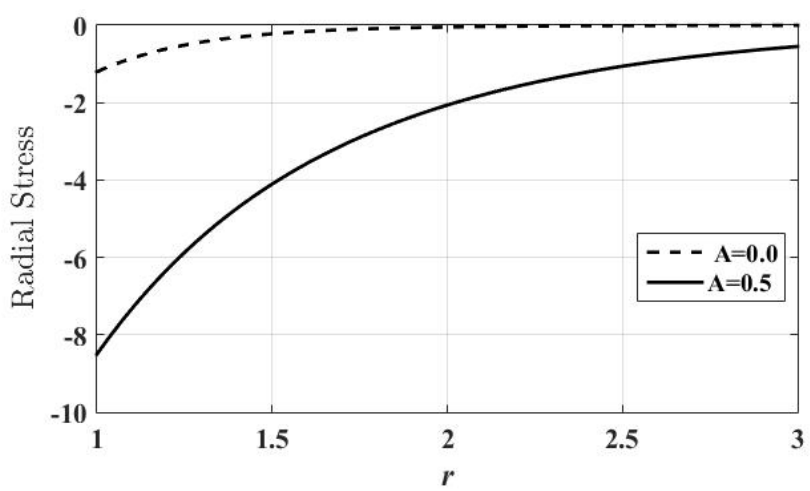

Fig. 4 Variation of Radial Stress $\sigma_{r r}$ with distance $\mathrm{r}$ for the coefficient of Ohm and Fourier laws

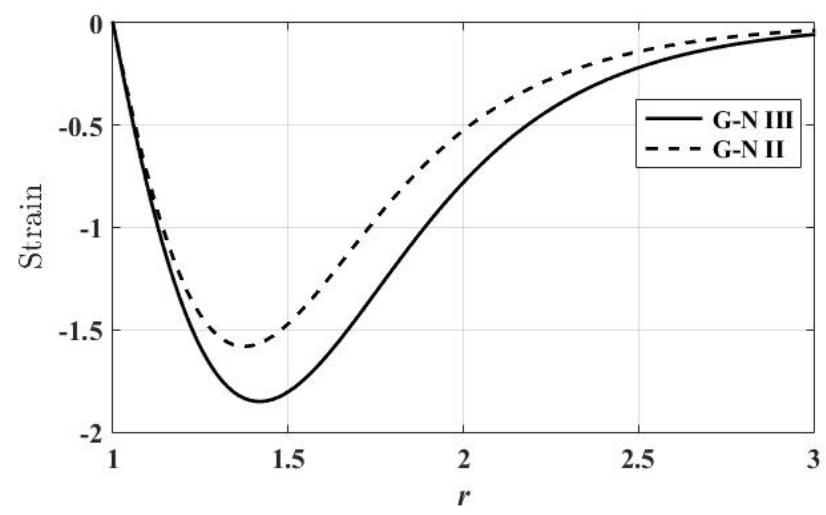

Fig. 5 Variation of Strain e with distance $r$ for two theories

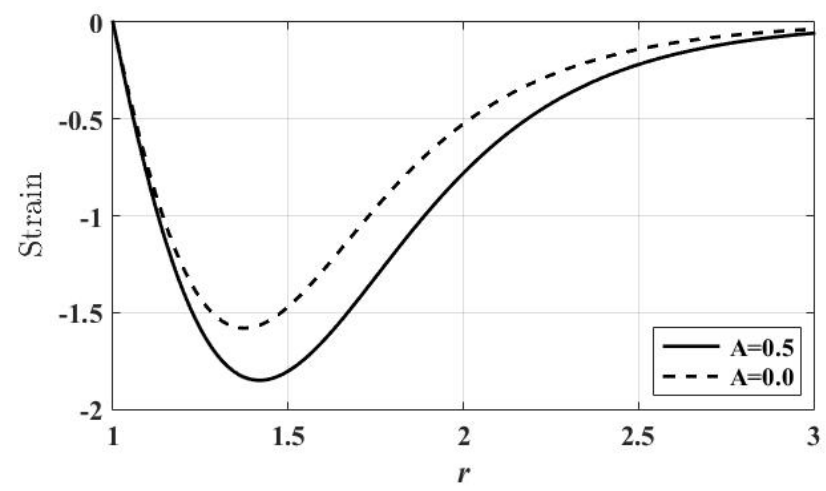

Fig. 6 Variation of Strain e with distance $r$ for the coefficient of Ohm and Fourier laws

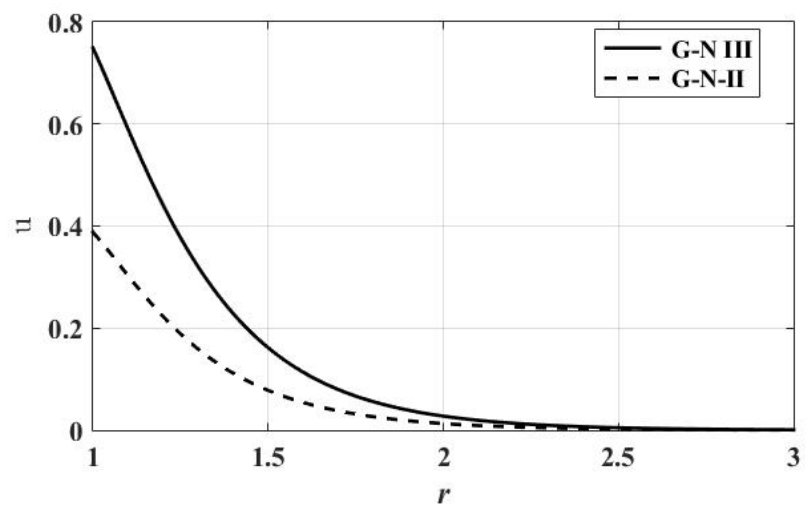

Fig. 7 Variation of Displacement $u$ with distance $r$ for two theories

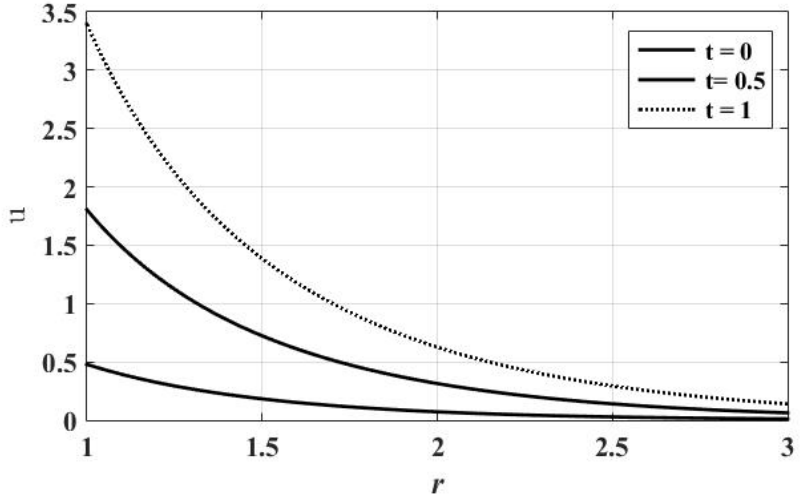

Fig. 8 Variation of Displacement $u$ with distance $r$ for different value of time $t$

Fig. 2 shows variation of temperature $\theta$ for the coefficient of Ohm and Fourier laws. It is noticed that the modified Fourier and Ohm laws influence is significant, the temperature in the modified model records value higher than these in the old model. Fig. 3 is plotted to show the variation of the radial stress $\sigma_{r r}$ against $r$ for wide range of $r(1 \leq r \leq 10)$, at small time $(t=0.07)$, for two theories (G-N III) and $(\mathrm{G}-\mathrm{N}$ II). It is observed from this figure the magnitude of the radial stress is greater for (G-N III) model than (G-N II). It can be noted that the speed of propagation of stress is finite and coincide with the physical behavior of viscoelastic material. Fig.4. shows variation of radial stress for the coefficient of $\mathrm{Ohm}$ and Fourier laws. It is noticed that the modified Fourier and $\mathrm{Ohm}$ model effects on the radial stress by increasing their values.

Fig. 5 is plotted to show the variation of strain $e$ against $r$ for wide range of $(1 \leq r \leq 3)$, at small time $(t=0.07)$, for two theories (G-N III) and (G-N II). It is observed from this figure the magnitude of the strain is greater for (G-N III) model than (G-N II). It can be noted that the speed of propagation of strain is finite and coincide with the physical behavior of viscoelastic material. Also, we can see from this figure that the boundary condition (57) is satisfied. Fig. 6 shows variation of strain $e$ for the coefficient of Ohm and Fourier laws. It is noticed that the modified Fourier and Ohm laws influence is significant, the strain in the modified model records value higher than these in the old model.

Fig. 7 is plotted to show the variation of displacement $u$ against $r$ for wide range of $(1 \leq r \leq 3)$, at small time $(t=0.07)$, for two theories (G$\mathrm{N}$ III) and (G-N II). It is observed from this figure the magnitude of the displacement is greater for (G-N III) model than (G-N II). It can be noted that the speed of propagation of displacement is finite and coincide with the physical behavior of viscoelastic material. Fig. 8 shows the variation of displacement $u$ against $r$ for wide range of $r(1 \leq r \leq 3)$, for different values of time $(t=0.0, t=0.5, t=0.1)$. And we have noticed that, the time $t$ has significant effects on displacement $u$. The increasing of the value of $t$ causes increasing of the value of displacement $u$, and displacement $u$ vanishes more rapidly.

\section{CONCLUSIONS}

From the above discussion, one can reason that the new model of generalized magneto-thermo-viscoelasticity predicts new qualities for the temperature, displacement, stresses and strain. The impact of the temperature gradient at adequately low temperature may cause new sorts of magneto-thermo-viscoelastic wave with explicit stability properties. The expansion in the estimations of temperature might be clarified as the lost heat producing from the development of electric flow; this heat might be the fundamental motivation behind why the deformation of the medium will in general be ordinary and the magnetic field records esteems more prominent than the qualities in the old model. As indicated by this work, numerous specialists in the field of generalized thermo elasticity have applied Green-Naghdi hypothesis (III-II) for thermo 
elastic problem and not many of them can effectively applied for magneto thermo Viscoelastic issue. Right now conclude that the greatness of the every single physical amount is more noteworthy for (G$\mathrm{N}$ III) model than (G-N II). It very well may be noticed that the speed of spread of every single physical amount is limited and match with the physical conduct of Viscoelastic material.

\section{ACKNOWLEDGEMENTS}

The authors wish to acknowledge the approval and the support of this research study by the Project NO. SCI-2018-3-9-F- 7795 from the Deanship of Scientific Research in Northern Border University, Arar, KSA.

\section{NOMENCLATURE}

$C_{E} \quad$ Specific heat at constant strain

$e \quad$ Cubical dilatation

$\boldsymbol{E} \quad$ Electric displacement vector

$e_{i j} \quad$ Components of strain tensor

$\boldsymbol{F}_{\boldsymbol{i}} \quad$ Components of Lorentz body force

$\boldsymbol{h}$ Induced magnetic field vector

$\boldsymbol{H}$ Total magnetic intensity vector

$\boldsymbol{H}_{\boldsymbol{o}} \quad$ Initial uniform magnetic field

J Current density vector

$K \quad$ Thermal conductivity

$q_{i} \quad$ Components of heat flux vector

$R \quad$ Radius of the shell

$t \quad$ Time

$T_{o} \quad$ Reference temperature

$u_{i} \quad$ Components of displacement vector

Greek Letters

$\alpha_{o}, \alpha_{1} \quad$ Viscoelastic relaxation time

$\alpha_{t} \quad$ Coefficient of linear thermal expansion

$\gamma_{e}=\left(3 \lambda_{e}+2 \mu_{e}\right) \alpha_{t}$

$\gamma_{o} \quad\left(3 \lambda_{e} \alpha_{o}+2 \mu_{e} \alpha_{1}\right) \alpha_{t} / \gamma_{e}$

$\delta_{i j} \quad$ Kronicker delta

$\lambda_{e}, \mu_{e} \quad$ Lame elastic constants

$\mu_{o} \quad$ Magnetic permittivity

$\theta \quad$ Temperature increment

$\rho \quad$ Density

$\sigma_{i j} \quad$ Components of stress tensor

\section{REFERENCES}

Amin, M.M., El-Bary, A.A., and Haitham atef, .M, 2018, "Effect of viscous fractional parameter on generalized magneto thermoviscoelastic thin slim strip exposed to moving heat source," Mater. Focus 7, 814- 823 .

https://doi.org/10.1166/mat.2018.1591

Biot, M.A, 1954, "Theory of stress-strain relations in anisotropic viscoelasticity and relaxation phenomena, ” J. Appl. Phys. 25 ,13851391.

https://doi.org/10.1063/1.1721573

Biot, M.A, 1955, "Variational principles in irreversible thermodynamics with application to viscoelasticity," Phys. Rev. $97,1463-1469$.

https://doi.org/10.1103/PhysRev.97.1463

Biot, M., 1956, "Thermo elasticity and Irreversible Thermo-Dynamics," J. Appl. Phys.27, 240 - 253. https://doi.org/10.1063/1.1722351

Bland, D.R., 1960, The theory of linear viscoelasticity, Pergamon Press, Oxford.

Drozdov, A.D., 1996, "A constitutive model in Thermoviscoelasticity," Mech. Res. Commun. 23, $543-548$. https://doi.org/10.1016/0093-6413(96)00055-9

Gurtin, M.E. and Sternberg. E., 1962, "On the linear theory of viscoelasticity,” Arch. Rat. Mech. Anal. 11 ,291-356.

https://doi.org/10.1007/BF00253942

Green, A. and Laws. N., 1972, "On the Entropy Production Inequality." Arch. Ration. Mech. Anal. 54.

https://doi.org/10.1007/BF00253395

Green, A.E., and Lindsay, K.A. 1972, “Thermo elasticity,” J. Elasticity. 2, 1-7.

https://doi.org/10.1007/BF00045689

Green, A.E., and Naghdi, P.M., 1991, "A re-examination of the basic postulate of thermo-mechanics," Proc. Roy. Soc. London, 432, 171-194. https://doi.org/10.1098/rspa.1991.0012

Green, A. E., and Naghdi, P.M., 1992, "An unbounded heat wave in an elastic solid,” J. Therm. Stress., 15, 253-264. 112.

https://doi.org/10.1080/01495739208946136

Green, A. E., and Naghdi. P.M., 1993, "Thermoelasticity without energy dissipation," J. Elasticity, 31, 189-208.

https://doi.org/10.1007/BF00044969

Illyushin's, A.A., and Pobedria. B.E, 1970, "Fundamentals of the mathematical theory of thermal viscoelasticity," Nauka, Moscow

Ismail, M.A., Shadia Fathi, El-Bary, A.A., and Youssef, M., 2020, "Generalized magneto- thermoelasticity and heat conduction on an infinite medium with spherical cavity, " Frontiers in Heat and Mass Transfer (FHMT), 14, 3. https://doi.org/10.5098/hmt.14.3

Koltunov, M.A., 1976, “Creeping and Relaxation,” Moscow.

Lord, H. and Shulman, Y., 1976, "A Generalized Dynamical Theory of Thermo elasticity,” J. Mech. Phys. Solid. 15, 299 - 309 https://doi.org/10.1016/0022-5096(67)90024-5

Morland, L.W, and Lee, E.H, 1960, "Stress analysis for linear viscoelastic materials with temperature variation, " Trans. Soc. Rheol. 4, $233-263$.

https://doi.org/10.1122/1.548856

Müller. 1976, Arch. Rat. Mech. Anal. 41.

https://doi.org/10.1159/000180709

Shuhubi , E., 1957, "Thermo elastic solid, in: A. C. Eringen (Ed.)," cont. phys II, Academic press, New York, ch.2.

Tanner, R.I., 1988, “Engineering Rheology,” Oxford University Press, Oxford.Therm. Stress., 15, 253-264. 